\title{
Utilization of Health Applications Among Patients Diagnosed with Chronic Diseases in Jazan, Saudi Arabia During the COVID-19 Pandemic
}

\author{
Ruwaym Alharbi' \\ Alanood Qadri' \\ Mashael Mahnashi' \\ Alshomokh Hakami ${ }^{1}$ \\ Bushra Darraj' \\ Jubran Shnaimer $\mathbb{D}^{2}$ \\ Ibrahim Gosadi $\mathbb{1}^{3}$ \\ 'Faculty of Medicine, Jazan University, \\ Jazan, Saudi Arabia; ${ }^{2}$ Family Medicine \\ Department, Jazan Health Affairs, \\ Ministry of Health, Jazan, Saudi Arabia; \\ ${ }^{3}$ Department of Family and Community \\ Medicine, Faculty of Medicine, Jazan \\ University, Jazan, Saudi Arabia
}

Purpose: Health applications are employed to aid patients with chronic diseases in effective long-term disease management. This investigation aims to measure the utilization of health applications among patients diagnosed with chronic conditions during the COVID-19 pandemic. Methods: This cross-sectional investigation was conducted in the Jazan region in Saudi Arabia between October 2020 and March 2021. Data were collected during interviews using a semi-structured questionnaire. The questionnaire collected items about the patients' demographics, morbidity, and use of electronic devices and health applications developed by the Saudi MoH. Logistic regression was used to assess factors associated with odds of reporting use of applications.

Results: A total of 658 patients were recruited. The mean age of the patients was 51 years, and $59 \%$ of them were females. The most frequently reported diagnosed condition was diabetes $(53.5 \%)$, followed by hypertension (42.7\%). Majority of the patients reported not using any health applications developed by the Saudi $\mathrm{MoH}(68.2 \%)$. Patients with higher educational levels and younger patients were more likely to report use of the applications (ORs: 4.2, 2.6 respectively). Comorbidity history and disease duration did not seem to influence the probability of reporting use of health applications ( $\mathrm{P}$-values $>0.05$ ).

Conclusion: Patients diagnosed with chronic diseases in Jazan had low utilization of health applications developed by the Saudi MoH. This indicates a need to encourage the use of these health applications, particularly among patients with chronic diseases and to consider use restriction among older patients with lower educational levels.

Keywords: health applications, chronic diseases, diabetes, hypertension, COVID-19, Jazan, Saudi Arabia

\section{Introduction}

Chronic diseases are major contributors to mortality and disability worldwide. According to the World Health Organization, chronic diseases are responsible for $71 \%$ of global mortality, with nearly 41 million individuals dying each year due to these conditions. ${ }^{1}$ Chronic diseases are defined as conditions that last for a year or more, require continuous medical attention, and may have an impact on quality of life. $^{2}$ Therefore, people affected with chronic diseases may be vulnerable to ramifications if their conditions are not well managed.

The use of health applications is one method employed to aid patients with chronic diseases in effective long-term disease management. As the global use of mobile devices has increased, many mobile applications have been developed to
Correspondence: Ibrahim Gosadi Faculty of Medicine, Jazan University, P.O. Box: 2349, Jazan, 8262I, Saudi Arabia Tel +966562I377II

Email gossady@hotmail.com 
assist patients with chronic conditions in disease management and the healthcare-seeking process. For example, a systematic evaluation of mobile applications used for diabetic self-management, identified 65 relevant applications with varying utility. ${ }^{3}$ Additionally, a review of mobile applications used for medication management found 328 related applications, with less than $18 \%$ of them developed by healthcare professionals or academia. Tabi et al concluded that there is a need for continuous assessment of these applications, either by a healthcare professional or by users, to ensure that these applications are improved, and trustworthy ones identified. ${ }^{4}$

The Saudi Ministry of Health (Saudi $\mathrm{MoH}$ ) is a major contributor to health services in Saudi Arabia. To improve healthcare services in the country, the ministry introduced multiple health applications that provide services such as appointment booking ${ }^{5}$ and telemedicine. ${ }^{6}$ Since the beginning of COVID-19 pandemic, more than half million cases affected with COVID-19 infection have been reported in Saudi Arabia. ${ }^{7}$ In response to the COVID-19 pandemic and associated social distancing measures, the Saudi $\mathrm{MoH}$ and the Saudi Data and Artificial Intelligence Authority have developed health applications for the provision of healthcare services linked to diagnosis, contact tracing, and monitoring of COVID-19 cases. ${ }^{8,9}$

Saudi Arabia has a high prevalence of chronic diseases. The Saudi Health Interview Survey is a 2013 nationwide survey conducted to determine the prevalence of chronic diseases and their risk factors. According to the survey, $28.7 \%$ of adult Saudis are obese, $15.2 \%$ have hypertension, $13.2 \%$ are diabetic, and $8.5 \%$ have hypercholesterolemia. Furthermore, the pervasiveness of these conditions is expected to rise due to the high prevalence of cases with borderline parameters related to blood pressure, blood glucose, cholesterol, or overweight. ${ }^{10}$ According to more recent International Diabetes Federation estimates, 4 million Saudi adults have diabetes. ${ }^{11}$

Several investigations were conducted in Saudi Arabia to assess the use of health applications developed by the Saudi $\mathrm{MoH}$ and to measure patients' satisfaction. An online survey that recruited 528 individuals to evaluate the effectiveness of the Seha Application in enhancing health care delivery indicated that less than half of the respondents $(47 \%)$ utilized the application. ${ }^{12}$ Other studies assessed the utilization of Saudi $\mathrm{MoH}$ health applications during the COVID-19 pandemic. An online survey developed to assess the use of the Mawid mobile application recruited a sample of 1993 users, with $82.1 \%$ finding the application easy to use and nearly $80 \%$ being highly satisfied with it. ${ }^{13}$ Furthermore, Abdel Nasser et al conducted another online survey in which 425 patients who used telemedicine services in Saudi Arabia between February and August 2020 reported different satisfaction levels with the services offered. Abdel Nasser et al reported that $53.4 \%$ of users were satisfied with the easy registration, while a smaller percentage were satisfied with the audio and video quality of the telemedicine session ( $40.1 \%$ and $41.9 \%$ respectively). ${ }^{14}$ Finally, in another online survey targeting patients who benefited from virtual clinic services in primary healthcare settings between March and July 2020, 439 patients were identified and factors associated with satisfaction were assessed, with male patients and those with lower education levels being more likely to be satisfied with the virtual clinic services. ${ }^{15}$

It should be noted that studies assessing Saudi MoHdeveloped health applications use an online approach to measure utilization and satisfaction level. However, this approach may neglect a significant proportion of the Saudi community who are suffering from a chronic disease and are unable to respond to online surveys. Additionally, the use of these health applications after the onset of the COVID-19 pandemic in Jazan, southwest of Saudi Arabia, among patients with chronic conditions is unknown. Thus, this study aimed to measure the utilization of Saudi $\mathrm{MoH}$ health applications during the period after the onset of the COVID-19 pandemic and their associated factors among patients diagnosed with chronic conditions via a community-based method.

\section{Methods \\ Context of the Study}

This was a cross-sectional investigation conducted between October 2020 and March 2021 that targeted patients diagnosed with chronic diseases who lived in Jazan, southwest of Saudi Arabia. The Jazan Health Ethics Committee granted ethical approval for the study (approval number 2032 dated September 20, 2020). In cases when no recruiting took place at a specific healthcare setting, a community-based approach was utilized to find subjects. This study was conducted in accordance with the Declaration of Helsinki.

\section{Data Collection Tool}

A semi-structured questionnaire that was completed during phone interviews was used to collect data. The questionnaire contained both open-ended and closed-ended 
questions. It was developed to assess data about the patients' demographics, morbidity, and use of electronic devices and health applications developed by the Saudi MoH.

Demographic data included the patients' age, gender, education level, social status, and area of residence. Morbidity-related items cover the type of disease, duration since diagnosis, history of comorbidities, and location of follow-up for management of their condition. Items connected to the use of Saudi MoH health applications comprised the name of the health applications used, type of services used, and difficulties in using the named health applications.

A consultant in epidemiology and a specialist in family medicine assessed the developed questionnaire's content and its ability to measure items needed to answer the study's research question. Additionally, a pilot study conducted interviews with ten patients from the targeted population to test the questions' clarity and the time needed to complete the interview. The interviews were performed by trained medical students who had attended a workshop on how to conduct the study to reduce the occurrence of measurement bias.

\section{Data Collection Process}

This study targeted patients diagnosed with diabetes, hypertension, dyslipidemia, cardiovascular diseases, asthma, cancers, thyroid disorders, inherited blood disorders, and osteoporosis. Patients identified as children, adults without chronic diseases in the Jazan region during the study period, and those who showed interest to participate but did not complete the interview were excluded. The subjects were recruited through several steps. Patient identification and approach were ensured via a poster designed to advertise the study. To target the population of interest, the poster was delivered to different primary care settings in the region and promoted on social media to increase response rates. The poster provided information about the study and the research team's contact details. Patients who were interested in participating were able to contact the research team directly, which facilitated the scheduling of phone interviews. Since data collection was performed via phone interviews, verbal informed consent was obtained before initiation of the interviews.

Phone interviews were employed as a data collection method to limit the risk of COVID-19 infection among the researchers and study participants. Patients who completed the recruitment process were advised to advertise the study to their relatives, neighbors, and friends and invite them to participate to increase the response rate and the demographic variability of the study's sample. It was noted that respondents' invites to their acquaintances enabled the recruitment of many patients, including illiterate ones. Given the impossibility of having a specific sampling frame to allow random sampling, this investigation relied on purposeful, convenient, non-random sampling to meet the required sample size.

Given that utilization of $\mathrm{MoH}$-provided mobile health applications was unknown at the time of designing the research protocol for this investigation, it was presumed that only $50 \%$ of patients with chronic diseases in the Jazan region used them. Using Epi Info's StatCal function and assuming a $50 \%$ prevalence, $5 \%$ margin of error, and $99 \%$ confidence level, the calculated sample size is 663 patients.

\section{Data Analysis}

Data were analyzed via the Statistical Package for Social Sciences (SPSS) version 25. For the binary and categorical data, we utilized frequencies and proportions to generate a summary. For continuous data, the mean and standard deviations (SD) were used to summarize the normally distributed data, while the median and interquartile range (IQR) values were used to summarize non-normally distributed data. In analyzing factors linked to the odds of using $\mathrm{MoH}$ health applications, logistic regression was calculated to estimate the odds of use based on the study demographics. Moreover, several categorical and continuous variables were grouped into binary variables to enable easier calculation of odds ratios (ORs) and reduce the number of empty cells. Selecting cut-off points to proceed with the binary classification was made via estimating means of continuous variables. For categorical variables including education level, occupation, marital status, and follow-up location, (a) patients with lower than secondary educational attainment were compared to those with secondary education or higher; (b) married patients were compared to those who were unmarried at the time of recruitment; (c) employed patients were compared to those who were unemployed, students, or housewives; and (d) patients who followed up at PHCs were compared to those who do so at hospitals or in the private sector. ORs were estimated to assess demographic and clinical variables associated with the use of Saudi $\mathrm{MoH}$ health applications among the recruited patients. A p-value of 0.05 was presumed as statistically significant for the estimated ORs. 


\section{Results}

Eight hundred thirty-six patients expressed an interest to participate in the study. One hundred and seventy-eight patients were excluded because they did not meet the inclusion criteria, such as not completing the interviews, being under the age of 18 , not being diagnosed with a chronic disease, or not residing in the Jazan region. The total number of participants in this investigation was 658 patients. The time needed to complete the interview varied between 7 and 10 minutes with each patient.

Table 1 explains the demographic characteristics of the patients. The mean age of the patients was 51 years, more than half of them were females (59\%), and nearly half had a high school education or higher. However, it was noted that nearly one-third of the patients were illiterate (186 patients). Most of the sample was married (70.4\%), and more than $60 \%$

Table I Demographic Characteristics of 658 Patients Diagnosed with Chronic Diseases in Jazan, Saudi Arabia

\begin{tabular}{|l|l|}
\hline Variables: & \\
\hline Age: mean [SD] & $5 \mathrm{I}[16]$ \\
\hline Gender: frequency [proportion] & \\
Male & $270[41 \%]$ \\
Females & $388[59 \%]$ \\
\hline Education: frequency [proportion] & \\
Elementary & $97[14.7 \%]$ \\
Intermediate & $52[7.9 \%]$ \\
High & $99[15 \%]$ \\
Bachelor & $219[33.3 \%]$ \\
Masters & $4[0.6 \%]$ \\
PhD & $1[0.2 \%]$ \\
Illiterate & $186[28.3 \%]$ \\
\hline Marital status: frequency [proportion] & \\
Married & $463[70.4 \%]$ \\
Divorced & $35[5.3 \%]$ \\
Widowed & $69[10.5 \%]$ \\
Single & $91[13.8 \%]$ \\
\hline Occupation: frequency [proportion] & \\
Student & $52[7.9 \%]$ \\
Governmental employee & $134[20.4 \%]$ \\
Private sector employee & $23[3.5 \%]$ \\
Military & $13[2.0 \%]$ \\
Housewife & $197[29.9 \%]$ \\
Unemployed & $186[28.3 \%]$ \\
Others & $53[8.1 \%]$ \\
\hline Residence type: frequency [proportion] & \\
Rent & $120[18.2 \%]$ \\
Owned & $538[81.8 \%]$ \\
\hline
\end{tabular}

were either unemployed, housewives, or students. Finally, many of the respondents owned their homes $(81.8 \%)$.

Table 2 illustrates morbidity data from the recruited sample of patients and the location of follow-up for management of their health conditions. The most frequently reported diagnosed condition was diabetes, followed by hypertension. Dyslipidemia was the least frequently reported diagnosed condition, followed by anemia. Only 186 patients (28.2\%) reported having more than one diagnosed condition. Many of the patients had an illness duration of fewer than 12 years following diagnosis. The most frequently reported healthcare facility for follow-up of health conditions was PHCs (46.7\%), followed by hospitals (33.5\%).

Table 3 describes the use of health applications among the study's sample. There were 116 patients $(17.7 \%)$ who reported not using any electronic devices. When asked if they use Saudi $\mathrm{MoH}$ health applications, many of the patients answered no (68.2\%). Among patients who did not use health applications, the most frequently reported reason was believing that these are not necessary for disease management, followed by not being aware of them. Sixty-six patients reported not using Saudi $\mathrm{MoH}$ health applications because of illiteracy, while 51 patients $(11.6 \%)$ did not know how to use them.

Table 2 Morbidity and Location of Follow Up of 658 Patients Diagnosed with Chronic Diseases in Jazan, Saudi Arabia

\begin{tabular}{|l|l|}
\hline Variables: & Frequency [Proportion] \\
\hline Diagnosed condition & \\
Diabetes & $352[53.5 \%]$ \\
Hypertension & $281[42.7 \%]$ \\
Asthma & $45[6.8 \%]$ \\
Anemia & $34[5.1 \%]$ \\
CVD & $42[6.4]$ \\
Thyroid disorders & $46[6.9 \%]$ \\
Dyslipidemia & $22[3.3 \%]$ \\
Osteoporosis & $35[5.3 \%]$ \\
Others & $26[3.9 \%]$ \\
\hline Patients with comorbidity: & $186[28.2 \%]$ \\
\hline Duration of the diagnosed condition*: & \\
Less than I2 years & $373[57.6 \%]$ \\
I2 years or more & $274[42.4 \%]$ \\
\hline Location of follow up*: & \\
PHC & $307[46.7 \%]$ \\
Hospital & $220[33.5 \%]$ \\
Private sector & $56[8.5 \%]$ \\
Other & $74[11.3 \%]$ \\
\hline
\end{tabular}

Note: * I missing cases for disease duration, one missing case for location of follow up. 
Table 3 Use of Electronic Devices and Saudi MoH Health Applications Among 658 Patients Diagnosed with Chronic Diseases in Jazan, Saudi Arabia

\begin{tabular}{|c|c|}
\hline Variables: & $\begin{array}{l}\text { Frequency } \\
\text { [Proportions }\end{array}$ \\
\hline \multicolumn{2}{|l|}{ Use of electronic devices } \\
\hline I do not use electronic devices & $116[17.7 \%]$ \\
\hline Mobiles only & 404 [61.6\%] \\
\hline Tablets or PCs & 136 [20.7\%] \\
\hline \multicolumn{2}{|l|}{ Do you use health applications } \\
\hline Yes & $209[31.8 \%]$ \\
\hline No & 449 [68.2\%] \\
\hline \multicolumn{2}{|l|}{ Reasons of not using health applications } \\
\hline Illiteracy & $66[15 \%]$ \\
\hline Believing health applications are not necessary & $192[43.5 \%]$ \\
\hline Not knowing how to use the applications & $51[11.6 \%]$ \\
\hline Not aware about the existence of health applications & $96[21.8 \%]$ \\
\hline Technical difficulties & $22[5.0 \%]$ \\
\hline Other & $14[3.2 \%]$ \\
\hline \multicolumn{2}{|l|}{ Reported use of health applications } \\
\hline Patients reporting use of Mawid? & 119 [18.2\%] \\
\hline Patients reporting use of Sehaty? & $86[13.1 \%]$ \\
\hline Patients reporting use of Tawakalna? & $37[5.7 \%]$ \\
\hline Patients reporting use of Tetamman? & $14[2.1 \%]$ \\
\hline
\end{tabular}

When patients were asked to name the Saudi $\mathrm{MoH}$ health applications they utilize, the most often reported application was Mawid (18.2\%), followed by Sehaty (13.1\%). When asked if they have used Saudi MoH applications for follow-up consultations, 98 patients said yes, with most indicating no difficulty in using health applications for consultations. However, a small number of patients reported difficulties consulting with medical professionals via the health applications due to difficulty of use, consultation rejection, and prolonged wait times. Most patients who had consultations via the Saudi MoH health application claimed that the consultation was conducted by an $\mathrm{MoH}$ physician.

Table 4 illustrates the logistic regression findings of factors associated with the likelihood of using health applications among the recruited sample of patients. The strongest effect was detected to be with educational level (OR: 4.2); patients with higher educational levels are more likely to report their use of the applications. Additionally, the odds of reporting application use were higher among younger subjects (OR: 2.6), those who were employed (OR: 2.6), and patients who reported following up on their conditions in healthcare settings other than PHCs (OR: 1.9), all of which were statistically significant (p-value $<0.05$ ). It was noted that comorbidity history and disease duration since diagnosis did not seem to influence the probability of reporting use of health applications.

\section{Discussion}

This was a cross-sectional investigation undertaken in the southwest of Saudi Arabia to measure utilization of Saudi $\mathrm{MoH}$ health applications and its associated factors among patients diagnosed with chronic conditions. The most frequently reported conditions were diabetes or hypertension, with nearly one-third of the sample reporting comorbidities. The recruited sample of patients showed limited use of health applications, with the most often reported reason being a belief that the applications were unnecessary. Most of the patients who used the health applications for followup consultations reported no difficulties. Factors influencing the odds of reporting use of the applications were age, educational level, employment, and location of follow-up.

The investigation's findings can be compared to multiple local and international studies. In a self-administered investigation by Rafiullah and David assessing the use of health applications among patients with diabetes in Saudi Arabia, it was reported that only $36 \%$ of their sample indicated the use of these applications. ${ }^{16}$ Nonetheless, it must be noted that utilizing a self-administered questionnaire is likely to exclude illiterate patients from their sample, which may partially explain why our sample had a lower proportion of patients who reported using the application (31.8\%).

Other, more recent, online surveys measuring the general population's use of $\mathrm{MoH}$ health applications revealed higher utilization rates. In a study assessing the use of the Seha application, $47 \%$ of respondents reported using it, which is significantly higher than our sample (13.1\%). ${ }^{12}$ Similarly, in another investigation that employed an online survey to evaluate the use of the Mawid application during the COVID-19 pandemic, only 204 (8\%) of the 2542 participants reported not using the application. ${ }^{13}$ This finding demonstrated higher utilization in comparison to our patient sample, where $81.8 \%$ of them reported not using the Mawid application. This marked variation can be partially explained by demographic and methodological differences, as our sample included patients who were older and illiterate, both of whom are unlikely to respond to online surveys. This suggests that using online surveys to measure utilization of health applications may yield greater utilization rates than other methods that can recruit subjects with limited abilities to respond to online, self- 
Table 4 Factors Associated with the Odds of Reporting Saudi MoH Health Applications Among 658 Patients Diagnosed with Chronic Diseases in Jazan, Saudi Arabia

\begin{tabular}{|c|c|c|c|c|c|}
\hline \multirow[t]{2}{*}{ Variables } & \multicolumn{2}{|c|}{$\begin{array}{l}\text { Use of Health Applications } \\
\text { Frequency [Proportions] }\end{array}$} & \multirow[t]{2}{*}{ ORs } & \multirow[t]{2}{*}{ 95\% Cls } & \multirow[t]{2}{*}{$P$ value } \\
\hline & No & Yes & & & \\
\hline $\begin{array}{l}\text { Age } \\
\text { Below } 51 \text { years } \\
51 \text { years or more* }\end{array}$ & $\begin{array}{l}179[57.4 \%] \\
270[78 \%]\end{array}$ & $\begin{array}{l}133[42.6 \%] \\
76[22 \%]\end{array}$ & 2.640 & $1.88-3.706$ & $<0.001$ \\
\hline $\begin{array}{l}\text { Gender } \\
\text { Male } \\
\text { Female* }\end{array}$ & $\begin{array}{l}173[64.1 \%] \\
276[7 \mid .1 \%]\end{array}$ & $\begin{array}{l}97[35.9 \%] \\
112[28.9 \%]\end{array}$ & 1.382 & $0.992-1.925$ & 0.056 \\
\hline $\begin{array}{l}\text { Education level } \\
\text { Less than high school education* } \\
\text { High school education or above }\end{array}$ & $\begin{array}{l}239[84.5 \%] \\
210[56.0 \%]\end{array}$ & $\begin{array}{l}44[15.5 \%] \\
165[44.0 \%]\end{array}$ & 4.268 & $2.917-6.245$ & $<0.001$ \\
\hline $\begin{array}{l}\text { Marital status } \\
\text { Married } \\
\text { Not married* }\end{array}$ & $\begin{array}{l}314[67.8 \%] \\
135[69.2 \%]\end{array}$ & $\begin{array}{l}149[32.2 \%] \\
60[30.8 \%]\end{array}$ & 1.068 & $0.744-1.532$ & 0.722 \\
\hline $\begin{array}{l}\text { Occupation } \\
\text { Employed } \\
\text { Unemployed/Students/ housewife* }\end{array}$ & $\begin{array}{l}120[53.8 \%] \\
329[75.6 \%]\end{array}$ & $\begin{array}{l}103[46.2 \%] \\
106[24.4 \%]\end{array}$ & 2.664 & $1.892-3.752$ & $<0.001$ \\
\hline $\begin{array}{l}\text { Residence type: } \\
\text { Rent } \\
\text { Owned* }\end{array}$ & $\begin{array}{l}77[64.2 \%] \\
372[69.1 \%]\end{array}$ & $\begin{array}{l}43[35.8 \%] \\
166[30.9 \%]\end{array}$ & 1.251 & $0.826-1.896$ & 0.290 \\
\hline $\begin{array}{l}\text { Comorbidity } \\
\text { One disease } \\
\text { More than one disease* }\end{array}$ & $\begin{array}{l}318[67.4 \%] \\
|3|[70.4 \%]\end{array}$ & $\begin{array}{l}154[32.6 \%] \\
55[29.6 \%]\end{array}$ & 0.867 & $0.599-1.254$ & 0.448 \\
\hline $\begin{array}{l}\text { Disease duration } \\
\text { Less than } 12 \text { years } \\
12 \text { years or more* }\end{array}$ & $\begin{array}{l}248[66.5 \%] \\
194[70.8 \%]\end{array}$ & $\begin{array}{l}125[33.5 \%] \\
80[29.2 \%]\end{array}$ & 1.222 & $0.872-1.713$ & 0.244 \\
\hline $\begin{array}{l}\text { Location of follow up } \\
\text { PHC* } \\
\text { Other }\end{array}$ & $\begin{array}{l}233[75.9 \%] \\
216[61.5 \%]\end{array}$ & $\begin{array}{l}74[24.1 \%] \\
135[38.5 \%]\end{array}$ & 1.968 & $|.403-2.76|$ & $<0.001$ \\
\hline
\end{tabular}

Note: *Reference groups.

administered surveys. A literature search for a comparable international investigation identified a US-based study that utilized an online survey in May 2020, which recruited 2210 respondents with chronic diseases, with $49 \%$ reporting engaging in telehealth. ${ }^{17}$ Additionally, similar to our findings, it was noted that in their patient sample, the aging population was less likely to benefit from telehealth services.

Our investigation did not seek to measure patient satisfaction with the use of the MoH-developed health applications. Nevertheless, our study identified factors related to the odds of using the applications, such as age, educational level, and employment status. These findings are comparable to those of a recent online survey by Alharbi et al, who recruited 439 patients who utilized virtual clinic services provided by primary healthcare settings in Riyadh, Saudi Arabia, between March and July 2020. Alharbi et al concluded that satisfaction with virtual clinics was higher among male patients, those with lower educational levels, and patients aged 18 to 59 years old. ${ }^{15}$ Another study found high levels of satisfaction among 145 patients who attended diabetes telemedicine clinics provided by King Saud University Medical City, in Riyadh, Saudi Arabia, as a response to the social distancing measures of the 
COVID-19 pandemic, with $86 \%$ indicating that they would use virtual clinics in the future. However, their study did not report an assessment of factors linked to satisfaction levels. ${ }^{18}$

The current study's findings reveal that older patients and those with less than a high school degree are less likely to use health applications developed by the Saudi $\mathrm{MoH}$. The onset of the COVID-19 pandemic and its accompanying social distancing measures induced limited access to healthcare facilities, especially during the curfew, which lasted from March to May 2020. ${ }^{19}$ The 449 patients' lack of access to healthcare services offered through health applications mandates advertising that emphasizes the importance of these applications and gives more consideration to elderly and less educated patients concerning the management of their conditions during similar circumstances. The notion of considering special requirements for older patients is similar to the findings of a systematic review conducted by Arnhold et al, who investigated the use of mobile applications for diabetics. They concluded that usability of applications for diabetes was either moderate or good among applications offering a small range of services, while applications with a larger number of services showed worse usability. ${ }^{20}$

Since the nature of our investigation involved the collection of data at a specific point in time, no data on the use of $\mathrm{MoH}$ health applications in Jazan before the COVID-19 pandemic is available. Nevertheless, it was expected that the utilization of services provided by health applications would increase. Several international reports indicated increased usage of telemedicine services during the pandemic. ${ }^{21,22}$ It was also implemented as a necessary option to limit the risk of COVID-19 transmission. ${ }^{23}$ However, our findings reveal low utilization among patients diagnosed with chronic diseases in the Jazan region, even after the onset of the pandemic.

\section{Strengths and Limitations}

This study has several strengths and weaknesses. One of its assets is the inclusion of patients with chronic diseases and varying demographic features in its sample, such as those with low educational levels and the elderly population. This enabled the provision of a more realistic estimate in comparison to other surveys utilizing an online self-administered approach. One of its limitations is its reliance on non-random sampling and recall of patients without access to usage data, either from the $\mathrm{MoH}$ or health care providers.

\section{Conclusion}

Our investigation found that patients diagnosed with chronic diseases in Jazan had low utilization of health applications developed by the Saudi MoH. In comparison to other groups, older patients and those with low education levels were more likely to report low utilization. When asked why they were not utilizing the applications, most believed that these were not important for seeking healthcare. More effort should be employed to encourage the use of these health applications, particularly among patients with chronic diseases. Future investigations are needed to discern specific factors influencing the usage and satisfaction level of health application users in the region. Furthermore, it is recommended that research be conducted to determine whether the limited use of health applications during the pandemic affected the quality of healthcare services provided and overall disease management. Finally, the restricted use of health applications among older patients with limited education levels indicate a need to establish initiatives aiming to increase use of health applications among this group of patients. These initiatives can involve providing educational programs to orient the patients about use the applications. In addition to orienting the patients, these orientation efforts can involve family members who are taking care of the patients. Additionally, utilization of voice-assisted commands, or providing designated calling services to the applications for patients whom are illiterate or unable to read may aid in better utilization of the health applications.

\section{Author Contributions}

All authors made a significant contribution to the work reported, whether that is in the conception, study design, execution, acquisition of data, analysis and interpretation, or in all these areas; took part in drafting, revising or critically reviewing the article; gave final approval of the version to be published; have agreed on the journal to which the article has been submitted; and agree to be accountable for all aspects of the work.

\section{Funding}

This research did not receive any specific grant from any funding agency.

\section{Disclosure}

The authors report no conflicts of interest for this work. 


\section{References}

1. World Health Organization. Noncommunicable diseases: key facts; 2018. Available from: https://www.who.int/news-room/fact-sheets /detail/noncommunicable-diseases. Accessed July 16, 2021.

2. National Center for Chronic Diseases Prevention and Health Promotion. About chronic diseases; 2021. Available from: https://www.cdc.gov/ chronicdisease/about/index.htm. Accessed July 16, 2021.

3. Brzan PP, Rotman E, Pajnkihar M, Klanjsek P. Mobile applications for control and self management of diabetes: a systematic review. J Med Syst. 2016;40(9):210. doi:10.1007/s10916-016-0564-8

4. Tabi K, Randhawa AS, Choi F, et al. Mobile apps for medication management: review and analysis. JMIR mHealth uHealth. 2019;7 (9):e13608. doi:10.2196/13608

5. Saudi Ministry of Health. (Mawid) service; 2021. Available from: https://www.moh.gov.sa/en/eServices/Pages/cassystem.aspx. Accessed July 16, 2021.

6. Saudi Ministry of Health. MOH: e-health application launched; 2017. Available from: https://www.moh.gov.sa/Ministry/MediaCenter/ News/Pages/News-2017-12-14-007.aspx. Accessed July 16, 2021.

7. MoH S. COVID-19 dashboard: Saudi Arabia; 2021. Available from: https://covid19.moh.gov.sa/. Accessed August 27, 2021.

8. Saudi Ministry of Health. (Tetamman) app; 2021. Available from: https://www.moh.gov.sa/en/eServices/Pages/Rest-assured.aspx. Accessed July 16, 2021.

9. Saudi Data and Artificial Intelligence Authoridy. About Tawakkalna; 2020. Available from: https://ta.sdaia.gov.sa/en/index. Accessed July 16, 2021.

10. Saudi Ministry of Health. Saudi health interview survey results. Available from: http://www.healthdata.org/sites/default/files/files/ Projects/KSA/Saudi-Health-Interview-Survey-Results.pdf. Accessed August 27, 2021.

11. International Diabetes Federation. IDF MENA members: Saudi Arabia; 2020. Available from: https://idf.org/our-network/regionsmembers/middle-east-and-north-africa/members/46-saudi-arabia. html. Accessed July 16, 2021.

12. Alharbi A, Alzuwaed J, Qasem H. Evaluation of e-health (Seha) application: a Cross-Sectional Study in Saudi Arabia. BMC Med Inform Decis Mak. 2021;21(1):103. doi:10.1186/s12911-021-01437-6

13. Alanzi TM, Althumairi A, Aljaffary A, et al. Evaluation of the Mawid mobile healthcare application in delivering services during the COVID-19 pandemic in Saudi Arabia. Int Health. 2021. doi:10.1093/inthealth/ihab018
14. Abdel Nasser A, Mohammed Alzahrani R, Aziz Fellah C, et al. Measuring the patients' satisfaction about telemedicine used in Saudi Arabia during COVID-19 pandemic. Cureus. 2021;13(2): e13382.

15. Alharbi KG, Aldosari MN, Alhassan AM, Alshallal KA, Altamimi AM, Altulaihi BA. Patient satisfaction with virtual clinic during Coronavirus disease (COVID-19) pandemic in primary healthcare, Riyadh, Saudi Arabia. J Family Community Med. 2021;28 (1):48-54. doi:10.4103/jfcm.JFCM_353_20

16. Rafiullah M, David SK. Health apps usage and preferences among Saudi patients with diabetes: a survey. Int J Clin Pract. 2019;73(5): e13345. doi:10.1111/ijcp.13345

17. Horrell LN, Hayes S, Herbert LB, et al. Telemedicine use and health-related concerns of patients with chronic conditions during COVID-19: survey of members of online health communities. J Med Internet Res. 2021;23(2):e23795. doi:10.2196/23795

18. Al-Sofiani ME, Alyusuf EY, Alharthi S, Alguwaihes AM, Al-Khalifah $\mathrm{R}$, Alfadda A. Rapid implementation of a diabetes telemedicine clinic during the coronavirus disease 2019 outbreak: our protocol, experience, and satisfaction reports in Saudi Arabia. J Diabetes Sci Technol. 2021;15 (2):329-338. doi:10.1177/1932296820947094

19. Gassem LB, Obaid R. Saudi Arabia back in business as coronavirus curfews end; 2020. Available from: https://www.arabnews.com/node/ 1693461/saudi-arabia. Accessed September 9, 2021.

20. Arnhold M, Quade M, Kirch W. Mobile applications for diabetics: a systematic review and expert-based usability evaluation considering the special requirements of diabetes patients age 50 years or older. J Med Internet Res. 2014;16(4):e104. doi:10.2196/jmir.2968

21. Centre for Disease Control. Trends in the use of telehealth during the emergence of the COVID-19 pandemic - United States, JanuaryMarch 2020; 2020. Available from: https://www.cdc.gov/mmwr/ volumes/69/wr/mm6943a3.htm. Accessed July 16, 2021.

22. Golinelli D, Boetto E, Carullo G, Nuzzolese AG, Landini MP, Fantini MP. Adoption of digital technologies in health care during the COVID-19 pandemic: systematic review of early scientific literature. J Med Internet Res. 2020;22(11):e22280. doi:10.2196/ 22280

23. Monaghesh E, Hajizadeh A. The role of telehealth during COVID-19 outbreak: a systematic review based on current evidence. $B M C$ Public Health. 2020;20(1):1193. doi:10.1186/s12889-020-09301-4
Patient Preference and Adherence

\section{Publish your work in this journal}

Patient Preference and Adherence is an international, peer-reviewed, open access journal that focusing on the growing importance of patient preference and adherence throughout the therapeutic continuum. Patient satisfaction, acceptability, quality of life, compliance, persistence and their role in developing new therapeutic modalities and compounds to optimize clinical outcomes for existing disease states are major areas of interest for the journal. This journal has been accepted for indexing on PubMed Central. The manuscript management system is completely online and includes a very quick and fair peer-review system, which is all easy to use. Visit http:// www.dovepress.com/testimonials.php to read real quotes from published authors. 